\title{
CASO 1 -2018: Epilepsia de ausencias juveniles y deterioro cognitivo: presentación de caso clínico
}

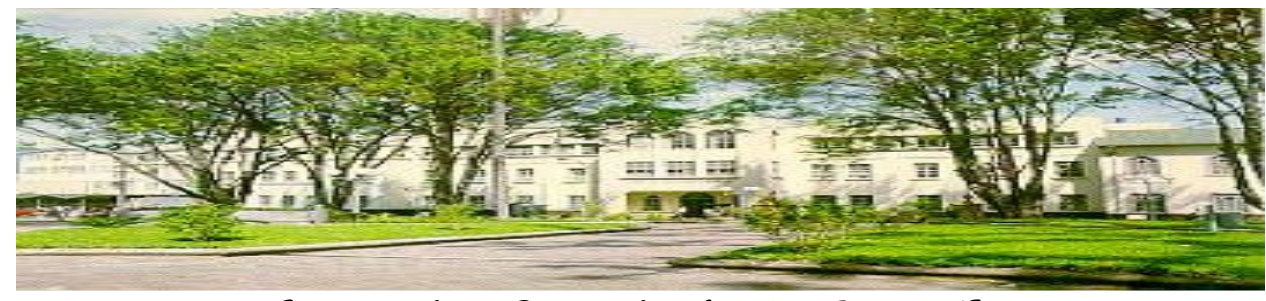

Haspital San quan de Dios, San Iosé, Casta Rica. Fundado en 1845

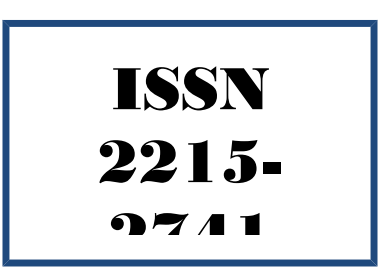

Recibido:

$07 / 12 / 2017$

Aceptado:

$15 / 01 / 2018$

Luis Eduardo Gómez Víquez. ${ }^{1}$

José Lee Chang Segura. ${ }^{2}$

Roberto Vargas Howell. ${ }^{3}$

\footnotetext{
${ }^{1}$ Médico General. Profesor de Neuroanatomía, Escuela de Medicina, Universidad de Costa Rica. Costa Rica. luis.gomezviquez@ucr.ac.cr

${ }^{2}$ Médico Residente de Neurología, Hospital Dr. Calderón Guardia, CCSS. Profesor de Neuroanatomía, Escuela de Medicina, Universidad de Costa Rica. Costa Rica.

${ }^{3}$ Médico Especialista en Neurología, Hospital Dr. Calderón Guardia, CCSS. Profesor del Posgrado de Neurología: Sistema de Estudios de Posgrado, Universidad de Costa Rica. Costa Rica.
}

\section{RESUMEN}

La epilepsia de ausencias juveniles (EAJ) es parte del espectro de las epilepsias genéticas generalizadas; su prevalencia se estima entre 0,2 y $2,4 \%$ de los pacientes con epilepsia. A continuación, se expone el caso de una paciente de 20 años quien fue evaluada por inicio en la adolescencia de una epilepsia de ausencias que asoció un deterioro cognitivo leve. Se discuten los principales determinantes del deterioro cognitivo en personas con epilepsia y se revisan las generalidades de la EAJ.

\section{PALABRAS CLAVE}

Epilepsis. Crisis de ausencias. Deterioro cognitivo. Síndrome epiléptico

\begin{abstract}
Juvenile absence epilepsy (JAE) is part of the spectrum of generalized genetic epilepsies, and its prevalence is estimated between 0.2 and $2.4 \%$ of patients with epilepsy. Next, it's exposed the case of a 20 -year-old patient who was evaluated for adolescence onset of an absence epilepsy that associated mild cognitive impairment. We discuss the main determinants of cognitive impair-
\end{abstract}


ment in patients with epilepsy and review the generalities of JAE.

\section{KEY WORDS}

Epilepsy. Absence crisis. Cognitive impairment. Epileptic syndrome.

\section{CASO CLÍNICO}

Paciente femenina de 20 años, con antecedente de retraso del desarrollo psicomotor leve, caracterizado por bajo rendimiento académico escolar; sin antecedentes de sufrimiento perinatal, trauma, infecciones de sistema nervioso central o crisis convulsivas durante la infancia. La paciente es referida con historia de que cuatro años previo al ingreso, empezó a presentar episodios descritos como crisis discognitivas, con poca respuesta al medio, con mirada fija y "rigidez" generalizada. Adicionalmente se recaba historia de episodios de movimientos estereotipados con ambas manos y caída de hombros de carácter mioclónico en ocasiones seguidas una de otra en periodos de 30 segundos y una frecuencia de unos 10 eventos por día. La paciente había iniciado medicación con valproato desde hacía 3 años, con mala respuesta terapéutica. Además, la paciente y sus familiares refieren un deterioro de su condición cognitiva base, mal rendimiento académico, así como trastornos del ánimo y labilidad emocional. La paciente había iniciado uso de fenobarbital, y fluoxetina dos semanas previo al ingreso con lo cual refería haber tenido mejoría sintomática. Se ingresó para estudio por epilepsia generalizada y deterioro cognitivo.

A la evaluación física se documentó buen estado general, con deterioro cognitivo leve (test de MOCA: 13 puntos). El resto de la exploración neurológica no presentó alteraciones. Se realizó electroencefalograma (EEG) en vigilia y sueño, previa supresión de sueño y de tratamiento farmacológico. Se reporta la presencia de puntasondas generalizadas breves y ritmo lento theta generalizado en vigilia, sin presentar episodios de crisis.

Se decidió someter a la paciente a monitoreo con video electroencefalografía. Durante el monitoreo se observó ritmo de fondo lento como descri- to en el EEG previo, con actividad interictal con descargas de puntas y ondas constantes; se documentaron 3 episodios clínicos de ausencias y mínima actividad mioclónica a nivel proximal del cuello en asociación a descargas de puntas y ondas generalizadas (ver imagen 1), que podrían corresponder con una epilepsia idiopática generalizada.

Complementariamente se realizó un estudio de resonancia magnética en el cual solo se aprecia leve polimicrogiria focal frontal derecha; el resto del estudio fue normal (ver imagen 2). Se complementó con estudios por enfermedades autoinmunes, inflamatorias, infecciosas, hemograma, bioquímica general: todos estos con resultados normales.

Se plantea como impresión diagnóstica un síndrome de epilepsia de ausencias juveniles, además de un deterioro cognitivo leve asociado a actividad interictal frecuente y retraso psicomotor de base. Se egresó a la paciente con tratamiento con valproato, fenobarbital y lamotrigina para seguimiento ambulatorio.

\section{Cuando hay una imagen, la descripción se coloca bajo la imagen}

Figura 1. Hernia de Amyand tipo 1. Fuente propia

\section{DISCUSIÓN Y ANÁLISIS DEL CASO}

\section{Introducción}

La epilepsia constituye la enfermedad neurológica adquirida más frecuente a nivel global, con una prevalencia estimada de alrededor del $1 \%$ de la población mundial. Los pacientes con epilepsia son una población particular por cuanto este padecimiento confiere un mayor riesgo de deterioro cognitivo en diferentes contextos y que se ven influenciados por diversos factores. En correlación al caso expuesto previamente, conviene revisar algunos aspectos respecto al deterioro cognitivo relacionado a epilepsia y las generalidades del síndrome de epilepsia de ausencias juveniles. 


\section{Disfunción cognitiva asociada a epi- lepsia.}

Es clara la asociación existente entre la presencia de crisis convulsivas y la mayor predisposición de un individuo a tener deterioro o retardo en el desarrollo de funciones cognitivas. Sin embargo, una de las principales dudas que se han planteado, es si realmente la presencia de crisis epilépticas per se es un detonante para generar este daño o si la asociación se debe a una causa común de fondo de ambos fenómenos. Al respecto, los estudios en modelos animales con crisis provocadas, han demostrado alteraciones metabólicas y celulares asociadas con atrofia hipocampal, alteraciones en la neoneurogénesis y la organización sináptica que predisponen a una mayor susceptibilidad a nuevas crisis no provocadas y deterioro cognitivo relacionado directamente a la frecuencia de crisis $^{(1)}$. En particular, para este caso clínico, la alta tasa de descargas en el EEG interictal, es uno de los factores que se debe tomar en cuenta como determinante en el deterioro a nivel neurocognitivo de la paciente.

Los principales problemas neurocognitivos que afectan a los pacientes con epilepsia son el deterioro en la memoria, el enlentecimiento mental y los trastornos atencionales ${ }^{(1)}$. La probabilidad de hallar lesiones estructurales focales o difusas es mayor en pacientes que presentan bajo rendimiento académico, problemas psicológicos o sociales $^{(2)}$. Los déficits de memoria son más marcados en las epilepsias focales, siendo más evidentes en cuanto a lenguaje verbal cuando se afecta el lóbulo temporal dominante (en particular en la epilepsia temporal mesial) y del lenguaje no verbal (en el hemisferio no dominante). La atención $\mathrm{y}$ las funciones ejecutivas se ven afectadas en epilepsias que afectan el lóbulo frontal (ya sea de manera primaria o por extensión) ${ }^{(3)}$. En el caso expuesto, pese a que en el momento que se abordó no se realizaron mediciones psicométricas, es probable que un problema a nivel atencional pudiera ser el causante del bajo rendimiento académico, principal molestia a nivel cognitivo de la paciente.

Otros factores que afectan la esfera cognitiva en los pacientes portadores de epilepsia y que no deben dejarse de lado al abordarlos, son los trastornos psiquiátricos acompañantes. Se ha demostrado que las personas con epilepsia tienen un riesgo 4-5 veces mayor de padecer concomitantemente ansiedad o depresión respecto a la población general, dichos trastornos se atribuyen principalmente a la restricción en actividades sociales, baja autoestima y rechazo social ${ }^{(4)}$. Asimismo, esto favorece una mayor disfunción en cuanto a la memoria episódica, atención, velocidad de pensamiento y procesamiento y funciones ejecutivas, exacerbando aún más los déficits cognitivos producto propiamente de la epilepsia ${ }^{(4)}$.

Prácticamente todas las drogas antiepilépticas tienen efectos neuromoduladores y repercusión en funciones neurocognitivas y psicomotoras. Tanto el valproato como el fenobarbital son drogas que producen una disminución de leve a moderada en la capacidad psicomotora y la velocidad de pensamiento. En el caso del valproato, estos efectos no son dosis dependientes, e incluso a dosis bajas pueden presentarse ${ }^{(5)}$. En el caso expuesto existe historia de uso crónico de valproato, el cual, si bien es cierto tiene un perfil de efectos secundarios más favorable que el de otros fármacos, también puede influenciar el rendimiento cognitivo general.

\section{Síndrome de epilepsia de ausencias ju- veniles.}

La epilepsia de ausencias juveniles (EAJ) corresponde a un tipo de epilepsia idiopática generalizada con un rango de inicio típicamente entre los 10-17 años. Son predominantes las crisis de ausencias, durante las cuales pueden presentarse automatismos palpebrales, en cara y manos. El patrón electroencefalográfico generalizado de punta-onda $2.5-4,5 \mathrm{~Hz}$ es el esperado en este síndrome. Es frecuente la presencia de crisis tónicoclónicas (hasta en $80 \%$ de los pacientes) y la presencia durante las crisis de movimientos mioclónicos correspondientes a las descargas de punta onda o polipunta-onda características del síndro$m e^{(6)}$. La prevalencia de esta patología se estima entre un $0,2-2.4 \%$ entre los pacientes con epilep$\operatorname{sia}^{(7)}$. En una gran cantidad de casos, solo se llega a identificar la presencia de epilepsia debido a la ocurrencia de crisis tónico-clónicas, las cuales son más evidentes que los episodios de ausen- 
cias. Esto, conlleva al subdiagnóstico de la patología ${ }^{(7)}$.

El origen de este síndrome se presume es genético, aún sin determinarse mutaciones específicas asociadas. Clínicamente, a diferencia de la espilepsia de ausencias infantiles, en la EAJ las crisis clínicamente manifiestas, son mucho más infrecuentes, de mayor duración y de menor intensi$\operatorname{dad}^{(6)}$. Todas estas características encajan dentro del perfil del caso expuesto.

La epilepsia de ausencias juveniles, junto con la epilepsia mioclónica juvenil y la epilepsia de ausencias infantiles conforman el grupo de las epilepsias generalizadas genéticas (idiopáticas). Este tipo de síndromes, si bien es cierto, tienen un buen pronóstico en cuanto al control de las crisis como tales, comparten la característica de presentar comorbilidades de índole cognitivo y de comportamiento que se han correlacionado con disfunción a nivel del lóbulo frontal ${ }^{(8)}$.

Otra asociación importante que se ha hecho con este tipo de epilepsias es la mayor incidencia de patología neuropsiquiátrica, en específico trastorno ansioso, depresivo, psicosis y esquizofrenia ${ }^{(9)}$. Algunos estudios han demostrado comorbilidad de hasta $4 \%$ de trastorno ansioso y de $21 \%$ de trastornos depresivos en los pacientes con epilepsia de ausencias juveniles ${ }^{(8)}$.

Se ha demostrado que hasta un $15 \%$ de los pacientes con EAJ presentan algún retardo mental concomitante, reflejado en un menor grado de coeficiente intelectual y de desempeño en pruebas neurocognitivas. Se ha propuesto que las posibles causas de esta disfunción serían relacionadas a lesiones orgánicas como microdisplasia cortical, lesiones producto de las descargas ictales o interictales, así como en parte, a efectos secundarios de las drogas antiepilépticas. También cabe destacar que la severidad de estas afectaciones es directamente proporcional al tiempo desde el inicio de las crisis y la frecuencia y duración de las mismas ${ }^{(10)}$. En cuanto a los hallazgos de neuroimágenes del caso reportado, llama la atención las discretas anomalías a nivel de la corteza frontal derecha que, como ya se mencionó, pueden estar presentes en este tipo de síndromes y ser un factor al menos en parte contribuyente al déficit cognitivo de los pacientes afectados ${ }^{(8,10)}$.

El tratamiento de la EAJ, de manera similar a la epilepsia de ausencias infantiles, incluye el uso en primera línea de fármacos de amplio espectro como el ácido valproico y otros fármacos específicos como el etosuximide ${ }^{(6)}$. Como tratamiento asociado y de segunda línea, se puede recurrir al uso de la lamotrigina, la cual por un efecto de inhibición de corrientes de calcio de tipo $\mathrm{T}$ a nivel del tálamo, favorece el control de las crisis ${ }^{(6)}$.

\section{CONCLUSIONES}

La EAJ es parte del espectro de las epilepsias genéticas generalizadas, se caracteriza como un síndrome de inicio en la adolescencia de episodios de ausencias de baja frecuencia y actividad clínica más limitada respecto a otros síndromes como el de epilepsia de ausencias infantiles. La asociación entre epilepsia y déficit cognitivo está bien tipificada, y se encuentra influenciada por factores causales comunes, comorbilidades psiquiátricas y neurobiológicas concomitantes, control de crisis, fármacos, entre otros. La EAJ no está exenta de estos factores y, por lo tanto, deben considerarse en la neurogénesis de los posibles trastornos cognitivos asociados al síndrome.

\section{BIBLIOGRAFÍA}

1. Katarzyna M, Pitkanen A. Do seizures cause irreversible cognitive damage? Evidence from animal studies. Epilepsy \& Behavior. 2004;(5).

2. Greener M. Beyond seizures: understanding cognitive deficits in seizures. Progress in Neurology and Psychiatry. 2013.

3. Rijckevorsel K. Cognitive problems related to epilepsy syndromes. especially malignant epilepsies. Seizure. 2006;(15).

4. McCagh J. Epilepsy, psychosocial and cognitive functioning. Epilepsy Reseach. 2009; 1(86).

5. Aldenkamp A. Effects of antiepileptic drugs on cognition. Epilepsia. 2001; 42(1). 
6. Westover M et al. Pocket Neurology. Segunda ed. Massachusetts: Wolters Kluwer; 2016.

7. Jallon P, Latour P. Epidemiology of Idiopathic Generalized Epilepsies. Epilepsia. 2005; 46(9).

8. Nickels K, Zaccariello M, Hamiwka L, Wirrell E. Cognitive and neurodevelopmental comorbidities in childrn epilepsy. Nature Reviews Neurology. 2016; 1.

9. Kumar S, Tekkalki B, Mohapatra S, Saha R. Mental Health Perspectives of Epilepsy: focus on anxiety disorders. Delhi psychiatry journal. 2015; 18(1).

10. Pavone P, Bianchini R, Trifiletti Rea. Neuropsychological assessment in children with absence epilepsy. Neurology. 2001;(56).

\section{CONFLICTO DE INTERÉS Y/O AGRADECIMIENTOS}

Los autores declaran que no existió ningún conflicto de interés en el presente reporte. 


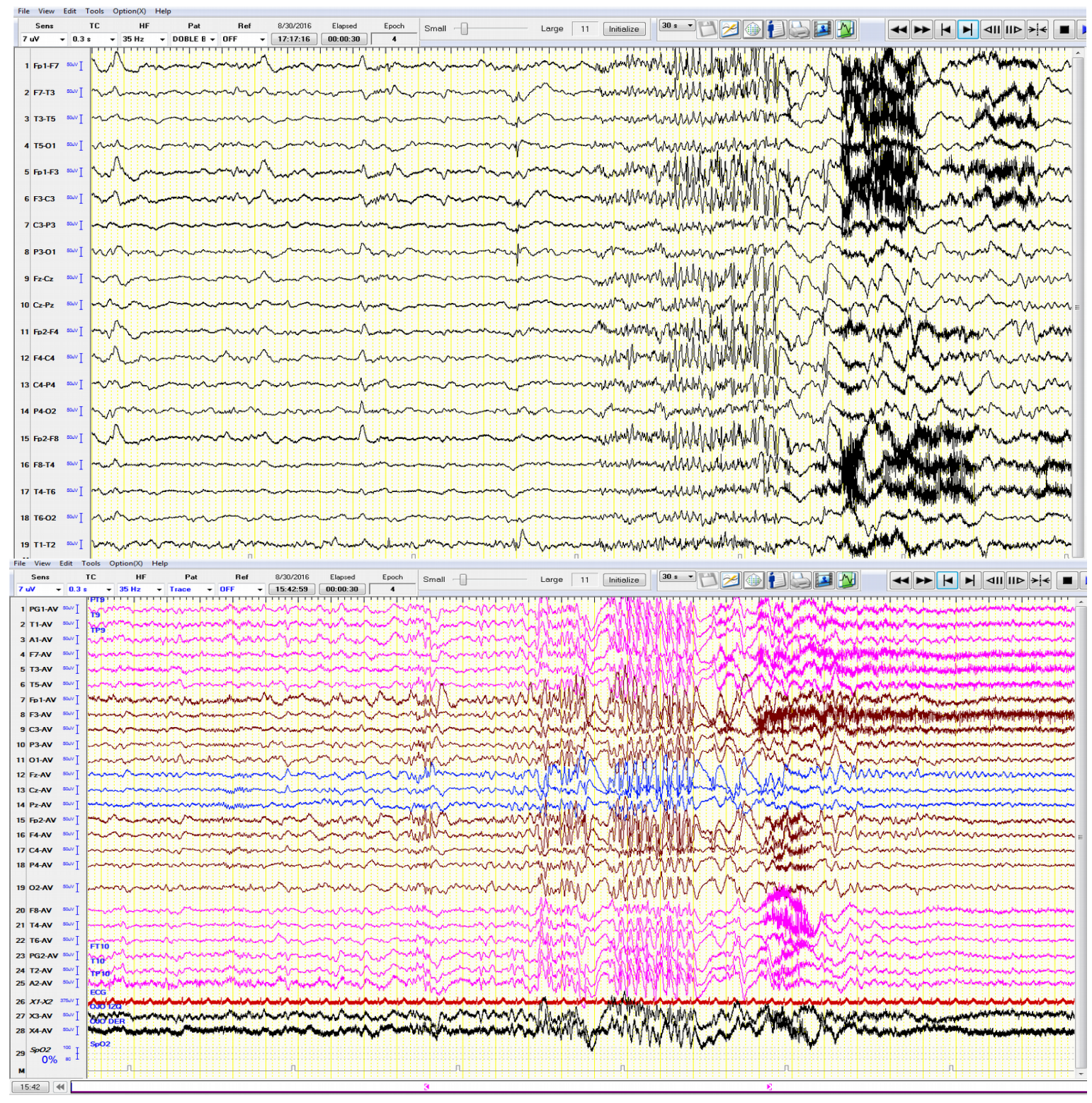

Figura 1. Trazo de EEG crítico, montajes referencial (superior) y bipolar (inferior). Episodio de ausencia asociado a contracciones leves a nivel de la musculatura cervical proximal. Se observan descargas de punta-onda generalizada de aproximadamente $3 \mathrm{~Hz}$.

Fuente: expediente clínico. 

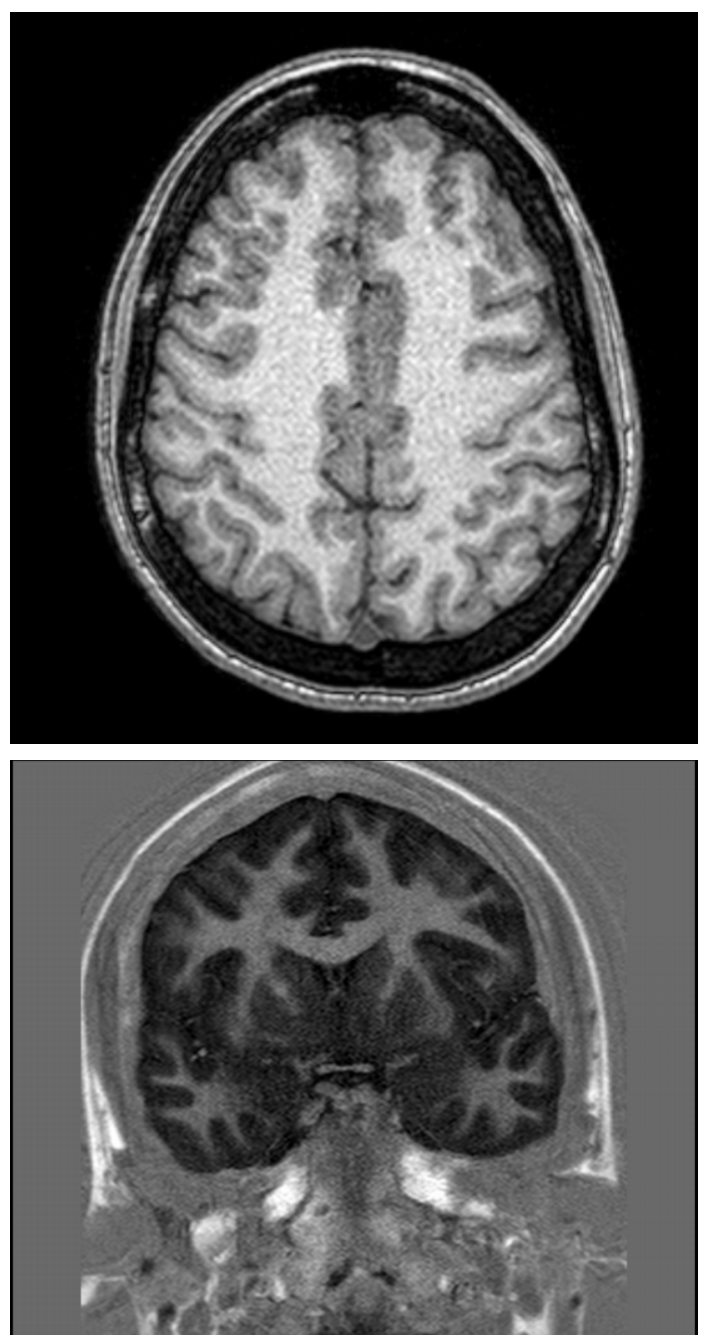

Figura 2. Resonancia magnética de la paciente donde se evidencia leve polimicrogiria frontal derecha. Corte axial T1 (parte superior) y coronal T1 inversion recovery. Fuente: expediente clínico.

Fuente: expediente clínico. 